\title{
SIGMA DELTA MODULATOR FOR BIOMEDICAL APPLICATIONS WITH REDUCED NONIDEALITIES
}

\author{
Bindu Patluri', Saxena .A.K' ${ }^{2}$, Dasgupta.$S^{3}$, \\ Semiconductor Devices and VLSI Technology group \\ Department of Electronics and Computer Engineering, Indian Institute of Technology, Roorkee, India \\ E-mail : 'bindu179@gmai.com²kumarfec@iitr.ernet.in ${ }^{3}$ sudebfec@iitr.ernet.in
}

\section{Abstract}

A low power second order sigma-delta modulator $(\Sigma \Delta \mathrm{M})$, for biomedical applications, has been presented in this paper. This can be used to digitize electrical biomedical signals like Electro-cardiogram (ECG), Electroencephalogram (EEG), and Electroretinogram (ERG). The proposed sigma delta modulator has been implemented by Switched Capacitor (SC) technique. Behavioral modeling of nonideal second order SC $\Sigma \Delta M$ was done to find out the integrator's opamp specifications to attain resolution of 10 bits. Integrator has been designed to minimize distortion and charge injection, and remove input dependent offset voltage. Dynamic latched comparator has been employed for power reduction. The feedback circuit has been designed for minimum usage of switches and capacitors. The simulated results of the proposed $\Sigma \Delta \mathrm{M}$ in standard CMOS $0.18 \mu \mathrm{m}$ technology, using Tanner tools gives $>10$ bits resolution and power consumption ranges from $50 \mu \mathrm{W}$ to $400 \mu \mathrm{W}$ with a $1.8 \mathrm{~V}$ power supply.

Key words: Biomedical application, SD Toolbox, Second Order Sigma-delta modulator.

\section{INTRODUCTION}

In biomedical instrumentation, biomedical signals such as Electrocardiogram (ECG), Electroencephalogram (EEG), Electrogastrogram (EGG), Electroretinogram (ERG) are analog in nature. The voltage and frequency ranges of some common biomedical signals are shown in Fig. 1 [1]. These biomedical signals are low frequency signals.

There is a necessity for biomedical signals to be converted into the digital domain for further analysis and signal processing using either conventional digital computers or special purpose digital signal processors (DSPs). As sigma-delta ADC can achieve the highest resolution for relatively low signal bandwidths [2], it is ideally suited for biomedical applications. In order to relax the anti-alias filter requirement and to minimize the digital noise coupling on chip, oversampled sigma-delta converters are used.

In analog circuit design, the voltage or current mode methodologies can be used for signal processing. In the voltage (current) mode design, information is encoded by voltage (current) or its derivatives. The Switched Capacitor (SC) technique has been extensively used in the voltage mode design while Switched Current (SI) techniques in the current mode design. Due to its ability to realize accurate signal processing function we have used SC technique.

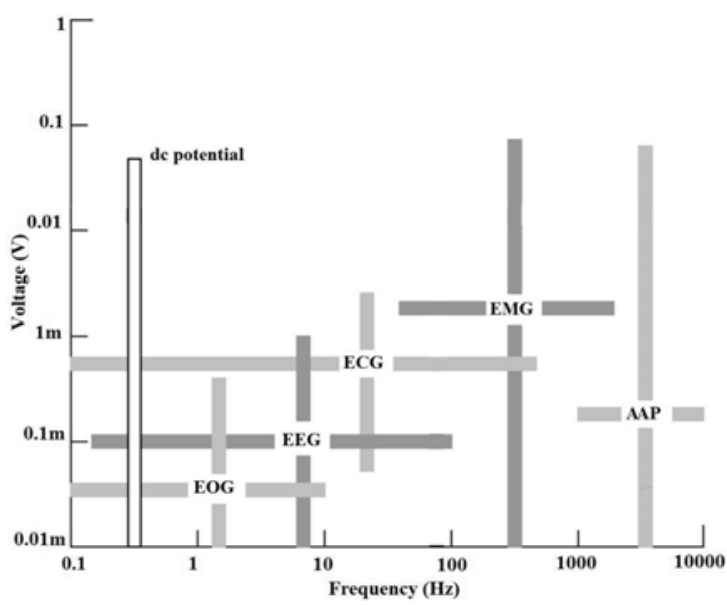

Fig.1 Voltages and Frequency Ranges of Some Common Bio-potential Signals [1]

In section II the behavioral modeling of $\Sigma \Delta \mathrm{M}$ has been presented. Section III discusses the circuit topologies for the design of low power low pass second order switched capacitor $\Sigma \Delta M$ to achieve a resolution $>10$ bits suitable to digitize biomedical signals such as ECG. Section IV presents the simulation results obtained.

\section{SYSTEM LEVEL ARCHITECTURE}

As the order of $\Sigma \Delta M$ goes beyond two, the system experiences potential instability while the usage of first order modulator to achieve required dynamic range results in large over sampling ratio (OSR) thus large power. A multibit quantizer produces harmonic distortion due to step-size mismatch [3]. Thus, second order modulator with 1-bit quantizer is used in our design. 
The architecture implementation of second order $\Sigma \Delta M$ is shown in Fig. 2. The values for integrators' gain $b_{1}$ and $b_{2}$ are used to protect integrators from saturation. MATLAB Simulink has been widely accepted to model and simulate $\Sigma \Delta \mathrm{M}$.

The output spectrum for the ideal modulator simulated using MATLAB and SIMULINK environment is shown in Fig. 3 for a sinusoidal input signal with $0.2 \mathrm{~V}$ amplitude and $200 \mathrm{~Hz}$ frequency.

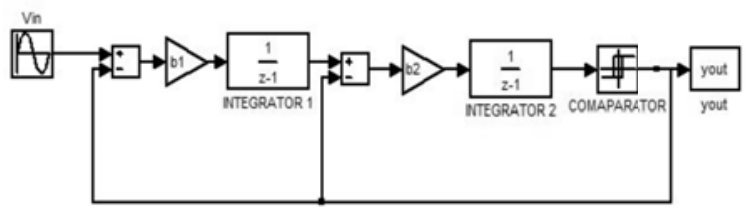

Fig.2 Ideal Second Order Sigma Delta Modulator

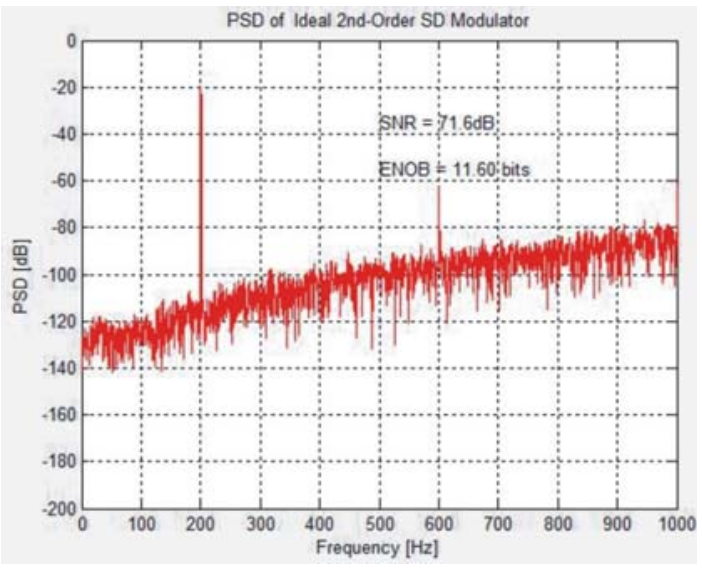

Fig. 3 PSD for lideal Second Order $\Sigma \Delta \mathrm{M}$

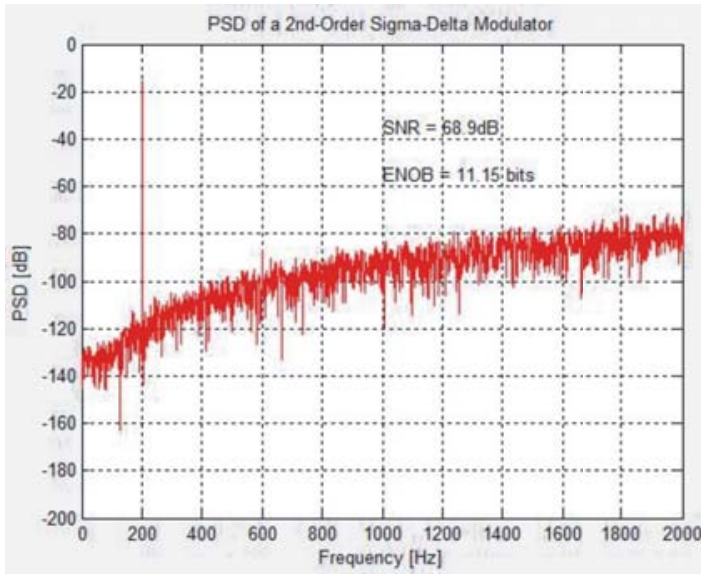

Fig. 4 PSD for Nonideal 2nd Order $\Sigma \Delta M$

But in real-time simulations, the modulator includes many non-idealities. The most important non-idealities such as sampling jitter, $\mathrm{kT} / \mathrm{C}$ noise, internal ADC/DAC's parameters mismatch and non-linearity, operational amplifier parameters (white noise, 1/f noise, finite dc gain, finite bandwidth, slew rate and saturation voltages) have been modeled in Simulink, using SD toolbox. The SD toolbox is a toolbox created to simulate Switched Capacitor (SC) $\Sigma \Delta M$ at behavioral level, within Simulink environment [4], [5].

And the behaviorally modeled nonideal second order modulator resulted in Power Spectral Density (PSD) as shown in Fig. 4. The Signal to Noise Ratio (SNR) for ideal modulator is $71.6 \mathrm{~dB}$ while the SNR for non-ideal modulator is $68.9 \mathrm{~dB}$.

\section{CIRCUIT LEVEL IMPLEMENTATION}

\section{A. Two Stage Opamp}

Switched capacitor integrator consists of opamp, switches and capacitors. A high gain opamp should be used for integrator to work properly. Hence, to meet the requirements of a high gain input stage and an output stage with high driving capabilities, a two stage opamp is used. Miller compensation technique is employed for its stability in closed-loop applications. Due to an unintentional feed-forward path through the Miller capacitor, a Right-Half-Plane (RHP) zero is resistor is inserted in series with the Miller capacitor to remove this RHP zero [6].

The differential pair in Fig. 6 is formed by n-channel MOSFETs, M1 and M2. The first stage gives a high differential gain and performs the differential to single ended conversion. The first stage of op-amp has the current mirror circuit formed by $p$-channel MOSFETs M3 and M4. The transistor M6 serves as a n-channel common source amplifier which is the second stage of opamp and is aided by current load M7. The bias of the opamp circuit is provided by M8 and $I_{B}[7]$, [8]. The opamp is designed to attain the specifications given in Table I.

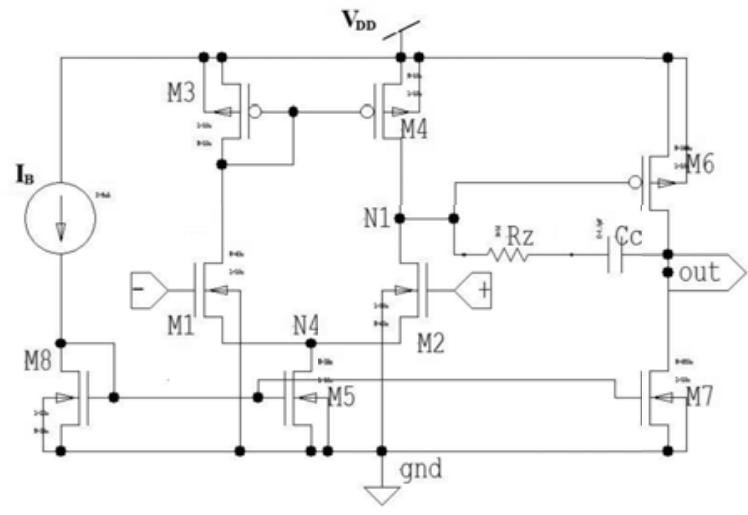

Fig. 5 Two Stage Opamp [7] 


\section{B. Parasitic Insensitive Switched Capacitor Integrator}

The single ended SC integrator which is parasitic insensitive is shown in Fig. 6. It is driven by two nonoverlapping clock phases P1 and P2. During the phase P1, integrator is in sampling mode and in phase $\mathrm{P} 2$, it is in integrating mode. The capacitors $\mathrm{C} 1, \mathrm{C} 2$ perform the integration, while $\mathrm{C} 3$ is an auxiliary capacitor used to compensate for the finite gain error and the offset voltage of the amplifier [9]. During phase 1, C1 is directly connected across the amplifier.

To minimize distortion, dc error and to keep dc offset low, clocks $\mathrm{P}_{1}, \mathrm{P}_{2}, \mathrm{P}_{1 \mathrm{a}}$ (slightly advanced wrt $\mathrm{P} 1$ ) are employed to turn off the switches near the virtual ground first.

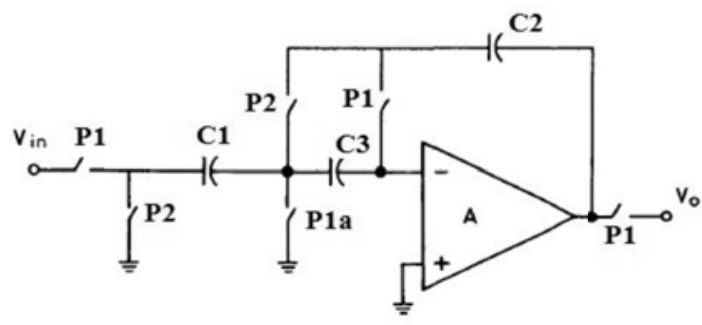

Fig. 6 Switched Capacitor Integrator [9]

By delaying clock $P_{1}$, for switch $S 1$ wrt clock $P_{1 a}$ for switch $S 3$, the input dependent offset voltage appearing at the output can be removed [7]. And switches connected to ground or virtual ground is realized using nmos switch to reduce the charge-injection effects [8].

\section{Comparator}

To reduce power, dynamic latch comparator is used which removes the need for pre-amplifying stage. [10] The 'Lewis-Gray' dynamic comparator shown in Fig. 7 was introduced by [11].

The operation of the comparator is as follows. When the clock signal is low, the transistors M9 and M12 are conducting and M7 and M8 are cutoff, which forces both differential outputs to $V_{D D}$ and no current path exists between the supply voltages. Simultaneously M10 and M11 are cutoff and the transistors M5 and M6 conduct. This implies that $\mathrm{M} 7$ and $\mathrm{M} 8$ have have a voltage of $\mathrm{V}_{\mathrm{DD}}$ over them. When the comparator is latched, the control signal goes up (clock $\left.=\mathrm{V}_{\mathrm{DD}}\right)$, which turns M7 and M8 on. Immediately after the switching moment, the gates of the transistors M5 and M6 are still at $V_{D D}$ and they enter saturation. The imbalance of the conductances of the left and right input branches formed by M1, M2, M5 and M3, $M 4, M 6$ determines which of the outputs goes to $V_{D D}$ and which to $0 \mathrm{~V}$. After a static situation is reached when clock=
$V_{D D}$, both branches are cutoff and the outputs preserve their values until the comparator is reseted again by switching latch to $0 \mathrm{~V}$.

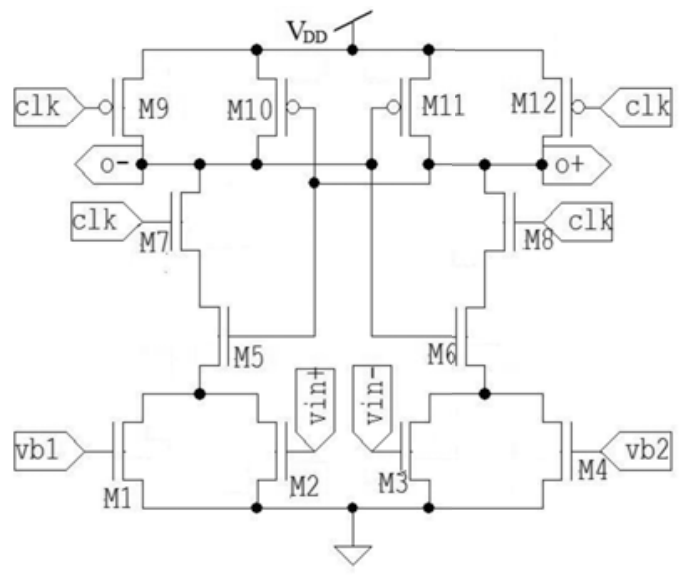

Fig. 7 Dynamic Comparator

The transistors connected to the input and reference M1-M4 are in the triode region and act like voltage controlled resistors. If no mismatch is present, the comparator changes its output when the conductances of the left and right input branches are equal. By denoting $W_{A}$ $=W_{1}=W_{3}$ and $W_{B}=W_{2}=W_{4}$ the input voltages when the comparator changes the state is given by

$V_{+i n}-V_{-i n}=\frac{W_{B}}{W_{A}}\left(V_{+r e f}-V_{-r e f}\right)$

By dimensioning of the transistors width $W_{A}$ and $W_{B}$, the threshold of the comparator can be adjusted to the desired level.

The comparator produces output of $V_{D D}$ when it is in reset mode. But in order to hold the previous value during reset an SR latch is used after passing the differential outputs through inverter.

\section{1-bitDAC and Summer Implementation}

The circuit implementation for feedback from comparator output to input of integrator through 1-bit DAC is achieved by implementing two input switched capacitor integrator, where one input is the input signal and second input is $-V_{\text {ref }}$ or $+V_{\text {ref }}$ depending on the output signal ' $y$ ' i.e. if $y=0,-V_{\text {ref }}$ is feedback and if $y=1, V_{\text {ref }}[12]$ as shown in Fig. 8.

\section{E. Complete Circuit Diagram of Second Order SDM}

Fig. 9 shows the complete second order $\Sigma \Delta M$. The feedback is designed to reduce number of switches and capacitors. During clock phase P1 the first integrator will be in sampling mode. During the clock phase P2, the first integrator is in integrating mode and the second one is in 
sampling mode. Now the comparator output is sensed at the beginning of the clock and latched. The output of comparator is used to provide feedback by applying $+\mathrm{V}_{\text {ref }}$ to second input of integrators, if output $y$ is high and $-V_{\text {ref, }}$ if $y$ is low. The feedback circuitry is designed to consume less area and power by reducing the number of switches and capacitors.

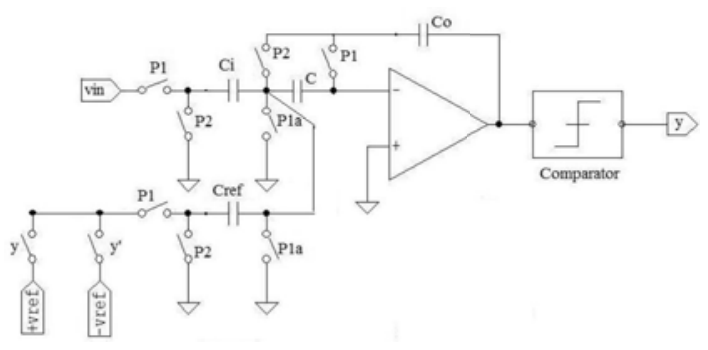

Fig. 8 First Order SD Modulator

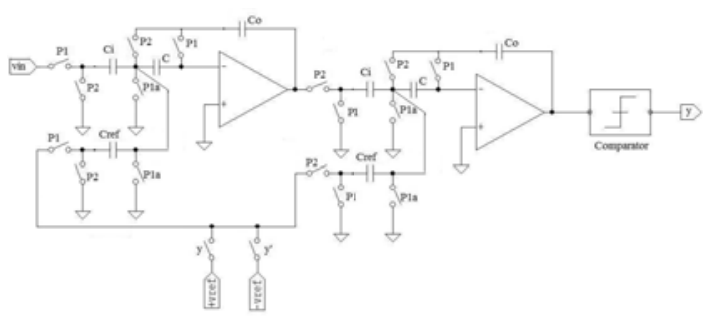

Fig. 9 Second Order SD Modulator

\section{RESULTS AND CONCLUSION}

From the behavioral modeling of the modulator, integrators' gain coefficients (b1 and b2), and opamp specifications are obtained, to achieve SNR of about $70 \mathrm{~dB}$ for input signal frequency of $200 \mathrm{~Hz}$ and OSR 64.

TABLE I

SPECIFICATIONS OF OP AMP

\begin{tabular}{cc}
\hline \hline Parameter & Simulated Result \\
\hline DC Gain $\left(A_{v o}\right)$ & $>45 \mathrm{~dB}$ \\
Unity Gain Bandwidth $(U G B)$ & $15 \mathrm{MHz}$ \\
Slew Rate $(S R)$ & $10 \mathrm{~V} / \mu \mathrm{s}$ \\
Phase Margin $(P M)$ & $>60 \mathrm{deg}$ \\
\hline \hline
\end{tabular}

n

technology node using power supply voltage of $1.8 \mathrm{~V}$. Tanner tools are used to simulate the above designed circuit. The total power consumed by the proposed $\Sigma \Delta \mathrm{M}$ ranges from $50 \mu \mathrm{W}$ to $400 \mu \mathrm{W}$.

Thus, a low power low pass $\Sigma \Delta \mathrm{M}$ has been designed with reduced nonidealities, so that dynamic range of $>10$ bits is met.

\section{ACKNOWLEDGMENT}

This research work is supported by 'Special Man Power Development in VLSI \& Related Softwares, Phase - II (SMDP-II)', Ministry of Information Technology, Government of India.

\section{REFERENCES}

[1] J. Goes, N. Paulino, H.Pinto, R. Monteiro, Bruno Vaz, S. Garção, Dec. 2005. "Low-Power LowVoltage CMOS A/D Sigma-Delta Modulator for Bio-Potential Signals Driven by a Single-Phase Scheme", IEEE Tran. Cicuits and Syatems-I, vol. 52, no. 12, pp. 25952604.

[2] Pervez M. Aziz, Henrik V. Sorensen and Jan Van Der Spiegel, Sep. 1996. "An Overview of SigmaDelta Converters-How a 1-bit ADC achieves more than 16-bit resolution", IEEE Signal Processing Magazine, vol.13, no. 1, pp. 62-84.

[3] Bernhard E. Boser, Bruce A. Wooley, Dec. 1988. "The Design of Sigma -Delta Modulation Analogto-Digital Converters", IEEE Journal Solid-State Circuits, vol. 23, no. 6, pp. 1298-1308.

[4] SIMULINK and MATLAB Users Guides, 1997. The MathWorks, Inc., Natick, MA.

[5] Category: Control and Systems Modelling, Control Design, SD Toolbox [Online]. Available: http://www.mathworks.com/matlabcentral/fileex change

[6] G. Palmisano and G. Palumbo, Mar. 1995. "An optimized compensation strategy for two-stage CMOS opamps," IEEE Trans. Circuits Syst. I, Fundam. TheoryAppl., no. 3, pp. 178-182.

[7] Phillip E. Allen and Douglas R. Holeberg, 2003. "CMOS Analog Circuits Design", $2^{\text {nd }}$ e/d, Oxford, New York,

[8] G. D. A. Johns and K. Martin, 1997. Analog Integrated Circuit Design, Wiley, New York.

[9] K. Nagaraj in Nagaraj, K., Vlach, J. , Viswanathan, T. R. and Singhal, K. , $9^{\text {th }}$ Oct. 1986. "Switched-Capacitor Integrator with Reduced Sensitivity to Finite Amplifier Gain," Electronics Letters, pp. 1102-1105, vol. 22, no. 21.

[10] Patheera Uthaichana, Ekachai Leelarasmee, Oct. 2003. "Low Power CMOS Dynamic Latch Comparators", IEEE TENCON, pp. 605-608, India, 14-17. 
[11] L.Sumanen, M. Waltari and K. Halonen, Dec. 2000. "A mismatch insensitive CMOS Dynamic Comparator", IEEE International Conference on Electronics, Circuits and Systems, vol. 1, pp. 3235, Lebanon, 17-20.

[12] I. Grech, J. Micallej, C.J. Debono, P. Malcovati, F. MalobertP, April 2001. "A 1V Second Order Sigma-Delta Modulator", J. Analog Integrated Circuits and Signal Processing, vol. 27, pp. 147-159.

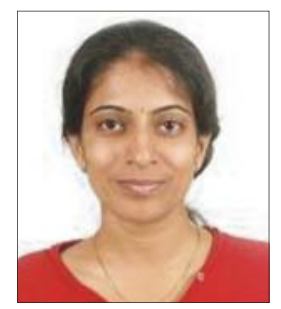

Bindu Patluri received the B.Tech degree in electronics \& communication engineering from JNTU, Hyderabad, India, in 2006 and the M.Tech degree in electronics \& computer engineering (semiconductor devices \& VLSI technology) from IIT Roorkee, India, in 2008. She worked on switched capacitor based sigma delta modulator, for M.Tech dissertation. Presently, she is working with Freescale Semiconductor, India as a Design Engineer.

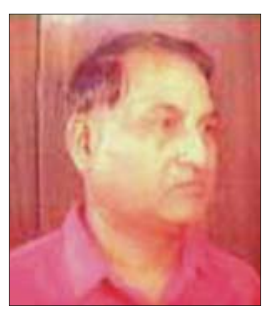

Dr. Saxena obtained Ph.D. from Department of Electronics and Electrical Engg.,UMIST/Sheffield University (UK) in 1975 and 1978, respectively as one of the two Government of India National Scholars. He is a Professor in Solid State Electronics and VLSI

Technology in IIT - Roorkee. The discovery of a level in GaAlAs is christened as 'Saxena's Deep Donor' by Philips Research Laboratory, Eindhoven (Netherlands). He is also a winner of INSA Young Scientist, Roorkee University Khosla Award Gold Medal, Kothari Scientific Research Institute Award, S. K. Mitra Memorial Awards (twice) of IETE and Bharat Excellence Award. He has published about 175 research papers in international journals and conference proceedings with very high citation index of about 775 so far..Dr. Saxena has supervised many Ph.D./M.E./M.Tech./M.Phil. theses in the area of VLSI design, metal-semiconductor ohmic and non-ohmic contacts, band structure and deep energy levels of GaAs, GaAlAs, GaP, InP, etc and quantum wells under pressure. He has also written AICTE sponsored nine volumes on the related subjects for working professionals. 